Research Article

\title{
Anti-Apoptotic Activity of Anthocyanins has Potential to inhibit Caspase-3 Signaling
}

\author{
Dewi Ratih Tirto Sari ${ }^{1,2}$, Anna Safitri ${ }^{1,3}$, James R. Ketudat Cairns ${ }^{4}$, Fatchiyah ${ }^{1,2 *}$ \\ ${ }^{1}$ Research Center of Smart Molecule of Natural Genetics Resources, Brawijaya University, Malang 65145, \\ Indonesia \\ ${ }^{2}$ Biology Department, Faculty of Mathematics and Natural Sciences, Brawijaya University, Malang 65145, \\ Indonesia \\ ${ }^{3}$ Chemistry Department, Faculty of Mathematics and Natural Sciences, Brawijaya University, Malang 65145, \\ Indonesia \\ ${ }^{4}$ School of Biochemistry, Institute of Science, Suranaree University of Technology, Nakhon Ratchasima 30000, \\ Thailand
}

Article history:

Submission June 2019

Revised September 2019

Accepted November 2019

*Corresponding author:

E-mail: fatchiya@ub.ac.id

\begin{abstract}
Caspase-3 is a biochemical marker for cell apoptosis. Several studies focused on exploring caspase inhibitor potential in natural compounds. Hence, in this study, we investigated the anthocyanins as anti-apoptotic potential activity through caspase-3 using molecular docking. Six types of anthocyanin were retrieved from PubChem database and caspase-3 protein was downloaded from Protein Data Bank. Anthocyanins and caspase3 protein were docked using HEX 8.0 program and visualized using Discovery Studio 4.1 software. The interaction among cyanidin-3-O-glucoside, delphinidin-3-O-glucoside, pelargonidin-3-O-glucoside, peonidin3-O-glucoside and petunidin-3-O-glucoside showed similar binding pattern on caspase-3 protein. All of them bind to BIR2 region and allosteric site of caspase-3, which are a crucial site for apoptosis regulation. Interestingly, cyanidin-3-O-glucoside, delphinidin-3-O-glucoside, pelargonidin-3-O-glucoside and peonidin-3-O-glucoside are tightly bind to BIR2 region and allosteric sites with hydrogen bond and hydrophobic interaction. Even though malvidin-3-O-glucoside also interacted with caspase-3 in BIR1, BIR2 and BIR3 regions. This study implies that cyanidin-3-Oglucoside, delphinidin-3-O-glucoside, pelargonidin-3-O-glucoside and peonidin-3-O-glucoside are more potent as anti-apoptosis through binding to caspase-3 than other anthocyanins. Although all anthocyanins have potential as an inhibitor of caspase- 3 protein and might have potential as anti-apoptosis. Further in-vitro and in-vivo studies are needed to confirm this experiment.
\end{abstract}

Keywords: Anthocyanins, Apoptosis, Caspase-3, In Silico Approach

\section{Introduction}

Apoptosis is an essential process for cellular degradation for cell growth and development [13]. The molecular marker of apoptosis is caspase, which is the proteolytic enzyme for protein or peptide. Caspase- 3 is one of the caspase family with the large and small domain structure. Caspase-3 has the activity as an effector for apoptosis. It is activated by phosphorylation in p38 at Ser150. The activated caspase- 3 will induce the cell leading to apoptosis [4-7].

Several studies have examined the caspase inhibitor both in-vivo and in-vitro studies. Peptidyl inhibitor for caspase has been reported to inhibit 
caspase-3 $[8,9]$. The z-DEVD-fmk, one of peptidyl inhibitor for caspase-3, has a potential activity as anti-apoptosis in rats brain tissue with acute cerebral infarction [10]. Furthermore, tetra-peptide aldehyde inhibitor AC-DEVD-CHO and AcDEVD-CMK also inhibit caspase-3 to prevent apoptosis pathway [11]. The X-linked inhibitor of apoptosis was reported to directly inhibit caspase3 in neuron cells of rat ischemic model. However, the peptidyl inhibitor for caspase- 3 is not preferred as a drug since the effect of it still being investigated. Besides, the peptidyl inhibitors show nonspecific caspase-3 inhibitor and low cell permeability in the cell [12]. Hence, some natural compounds have been explored in their anti-apoptosis activity. Some compounds have been identified as caspase inhibitor. They are rosmarinic acid, curcumin, luteolin, huperzine A, quercetin, resveratrol, bilobalide, EGCG, apigenin, berberine, and chitosan [13]. Flavonoid inhibits caspase-3 activity in MDA-MB-231 cells in in-vitro study [14].

Anthocyanin is the flavonoid derivate that as a bioactive compound in black rice [15, 16]. Black rice anthocyanins have been reported promoting some health benefits as anti-obesity $[17,18]$, antioxidant [19, 20], anti-inflammatory [21] and antiapoptosis [22, 23]. Black rice anthocyanins also induce cell proliferation and inhibit the apoptosis pathway [23]. Anthocyanin also promotes antiapoptotic effect on the human retinal pigment epithelial cells [24]. However, the mechanism is still unclear. This study focused to determine the function of anthocyanins as a human caspase- 3 inhibitor by in silico approach.

\section{Material and Methods \\ Ligand and protein preparation}

The 3D structures of black rice anthocyanins, cyanidin-3-O-glucoside (CID 12303221), delphinidin-3-O-glucoside (CID 443650), malvidin-3glucoside (CID 443652), pelargonidin-3-O-glucoside (CID 3080714), peonidin-3-glucoside (CID 443654), and petunidin-3-O-glucoside (CID 443651), were obtained from PubChem NCBI database. We used PyRx software to minimize their energy and convert the .sdf format into .pdb format [25]. The caspase-3 protein was retrieved from the RCSB Protein Data Bank (PDB ID: $1 \mathrm{nms}$ ) and was removed from water and ligand using Discovery Studio 4.1 program (http://3dsbiovia.com/products/).

\section{Molecular docking simulations}

The HEX 8.0 software was used in this study to predict the interaction and energy binding of anthocyanins (cyanidin-3-O-glucoside, malvidin-3-Oglucoside, peonidin-3-O-glucoside, petunidin-3-Oglucoside, pelargonidin-3-O-glucoside, and delphinidin-3-O-glucoside) and caspase-3 protein. The docking result was visualized using Discovery Studio 4.1 program (http://3dsbiovia.com/products/).

\section{Results and Discussion}

Cyanidin-3-O-glucoside bound to caspase-3 in Glu124, Lys137, Pro201, and Tyr195 (Figure 1A). Based on the 2D structure, cyanidin-3-O-glucoside-caspase-3 demonstrated some amino acid residues of caspase-3 with van der Waals (Figure 1A3). The Glu124, Lys137, Tyr195, and Pro201 were also identified in caspase-3 and delphinidin3-O-glucoside, pelargonidin-3-O-glucoside or peonidin-3-O-glucoside interactions (Figure 2, 4, and 5). Interestingly, cyanidin-3-O-glucoside and pelargonidin-3-O-glucoside had a similar active site when interacted with caspase-3. Some previous studies reviewed that cyanidin-3-O-glucoside and pelargonidin-3-O-glucoside differed on R1. Cyanidin-3-O-glucoside has - $\mathrm{OH}$ in R1, while pelargonidin-3-O-glucoside has $-\mathrm{H}$ in $\mathrm{R} 1$ [26, 27]. The structure of petunidin-3-O-glucoside is significantly different compared with cyanidin-3-O-glucoside and pelargonidin-3-O-glucoside. Petunidin-3-O-glucoside is a methylated anthocyanin in R3, while cyanidin-3-O-glucoside and pelargonidin-3-O-glucoside are non-methylated anthocyanins [26, 27, 28]. Interestingly, the interaction of petunidin-3-O-glucoside and caspase-3 took place in the same position as of caspase- 3 and cyanidin-3-O-glucoside or pelargonidin-3-O-glucoside interaction only at glucose group. Delphinidin-3-O-glucoside and peonidin-3-O-glucoside bound to caspase- 3 in the same active site.

There were three amino acid residues that have been detected in the malvidin-3-O-glucosidecaspase-3 interaction, namely Asp34, Arg238 and Arg241 (Figure 3-A2). Petunidin-3-O-glucoside bound to caspase-3 in the Glu124, Lys137, Asn141 and Tyr203 (Figure 6-A2). Those data indicated that cyanidin-3-O-glucoside, delphinidin3-O-glucoside, pelargonidin-3-O-glucoside, petunidin-3-O-glucoside and peonidin-3-O-glucoside interacted with caspase- 3 in the same pocket 


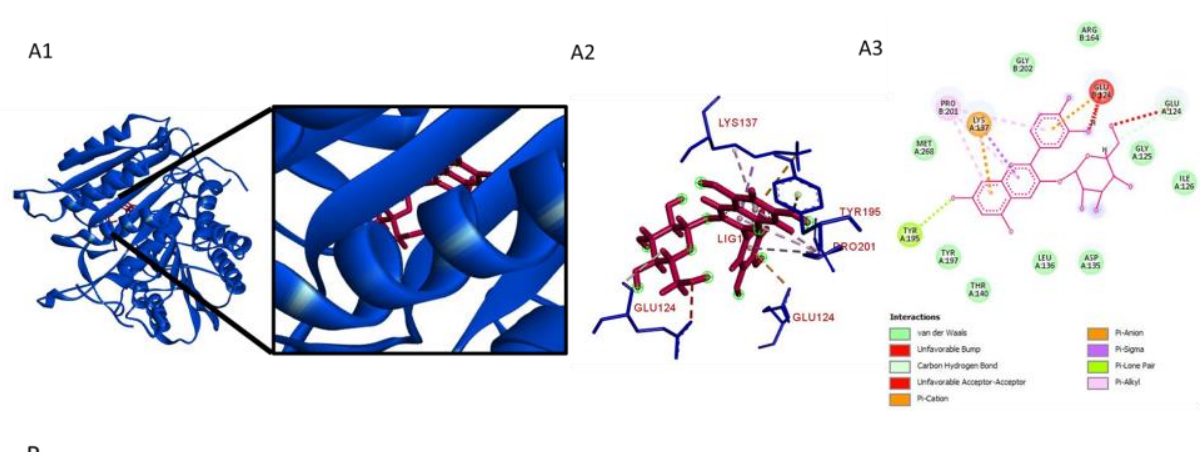

B
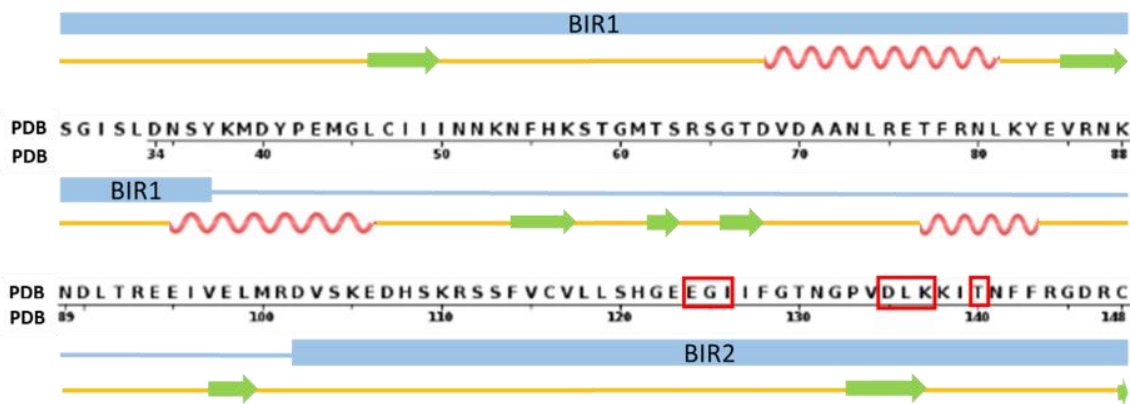

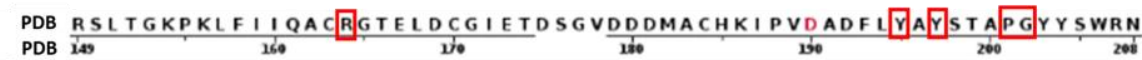
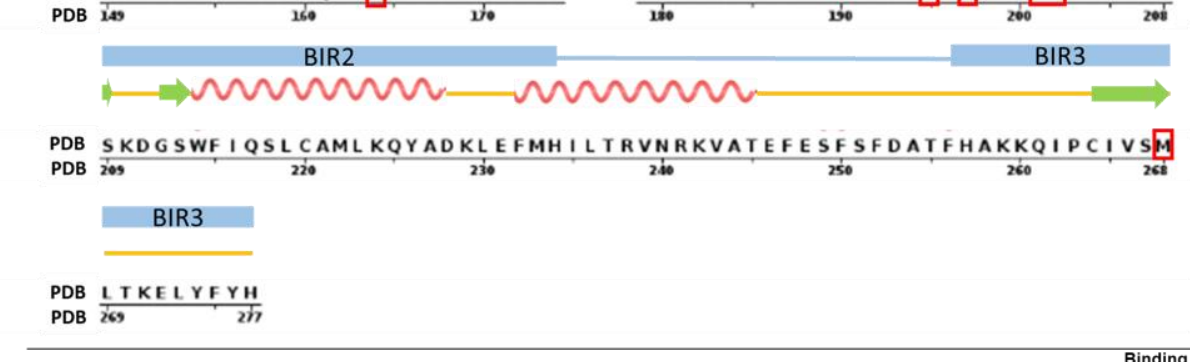

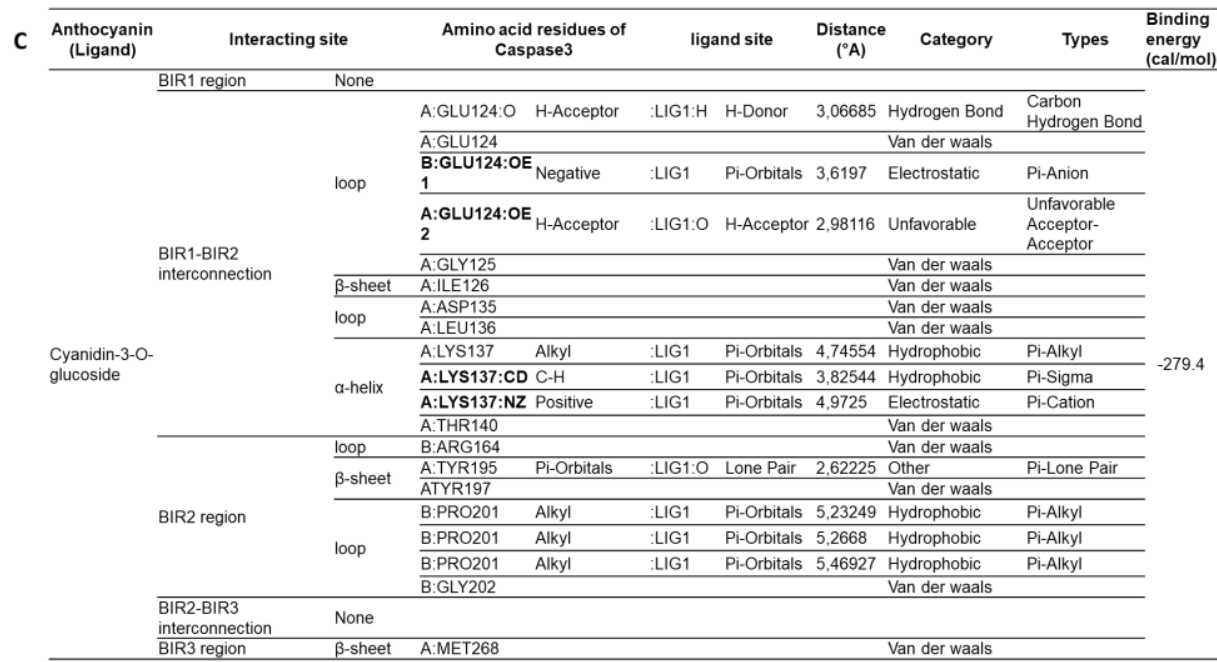

Figure 1. The interaction between cyanidin-3-O-glucoside and caspase-3. A1. Overview of cyanidin-3-O-glucoside-caspase-3 complex, A2. The 3D structure of cyanidin-3-O-glucoside-caspase-3 complex, A3. The 2D structure of cyanidin-3-O-glucoside-caspase-3 complex. Caspase-3 protein and cyanidin-3-O-glucoside were shown in blue and pink, respectively. B. Structure-based sequence of Caspase-3 domain interact with cyanidin-3-O-glucoside. The red box in the amino acid sequence indicates the binding site of anthocyanin-caspase-3. The secondary structure of caspase-3 is represented by the green arrow ( $\beta$ sheet), the red wave ( $\alpha$-helix), and the yellow line (loop). C. The binding site of cyanidin-3-O-glucoside into caspase-3. 


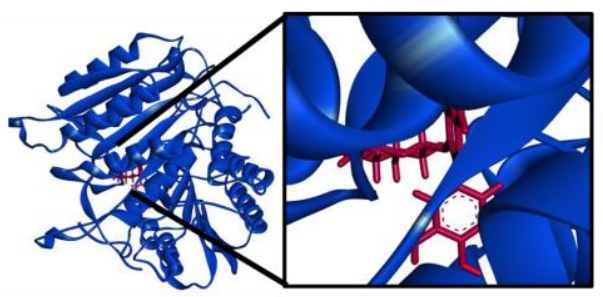

B

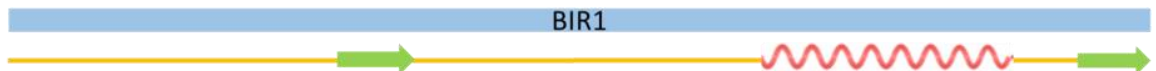

PDB SG I SLDNSYKMDYPEMGLCI I INNKNFHKSTGMTSRSGTDVDAANLRETFRNLKYEVRNK

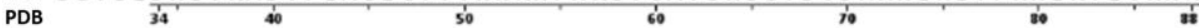

BIR1

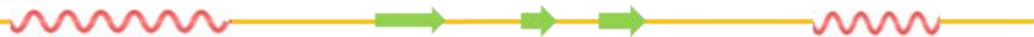

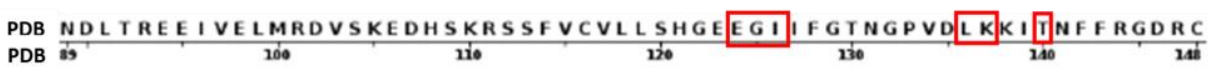

BIR2

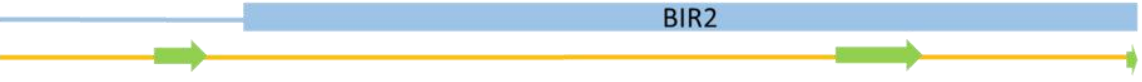

PDB RSLTGKPKLFI I QACEGTELDCG IETDSGVDDDMACHKI PVDADFL VA YSTAPGYYSWRN

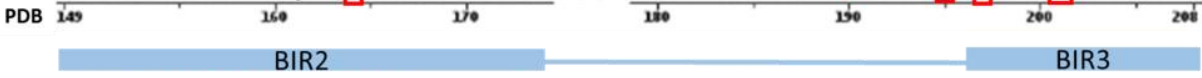

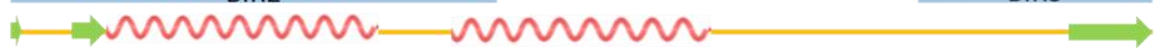

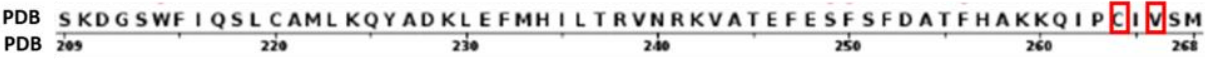

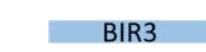

PDB LTKELYFYH

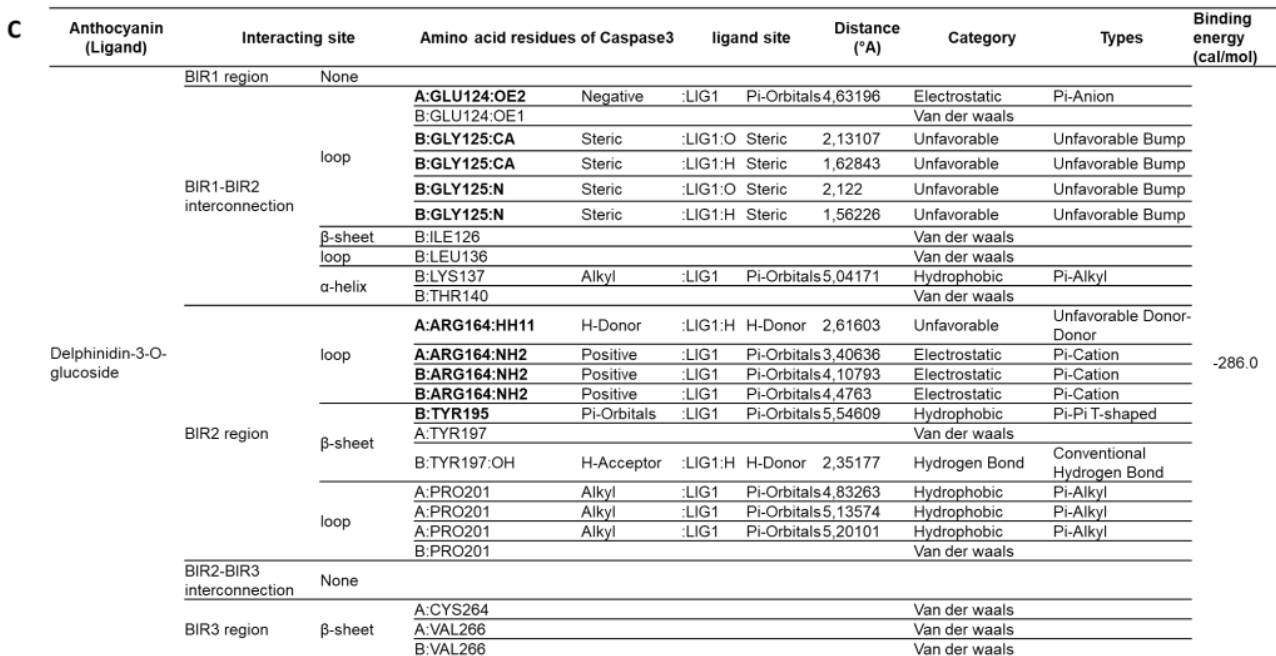

Figure 2. The interaction between delphinidin-3-O-glucoside and caspase-3. A1. Overview of the delphinidin-3O-glucoside-caspase-3 interaction. The 3D and 2D structure of the complex are presented in A2 and A3, respectively. B. The sequence of delphinidin-3-O-glucoside-caspase-3, the red box indicates the binding site of delphinidin-3-O-glucoside into caspase-3, the green arrow represents the $\beta$-sheet, the red wave is $\alpha$-helix and the yellow line represents the loop. The interaction between delphinidin-3-O-glucoside and caspase- 3 is demonstrated in C. 
A1

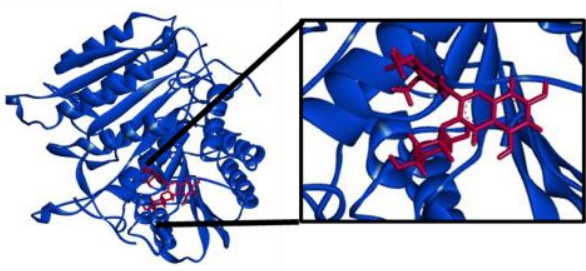

B

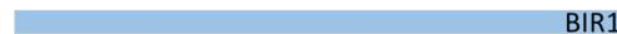

A3

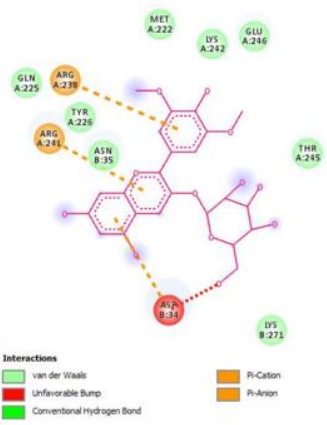

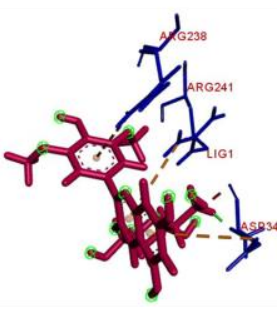

BIR1
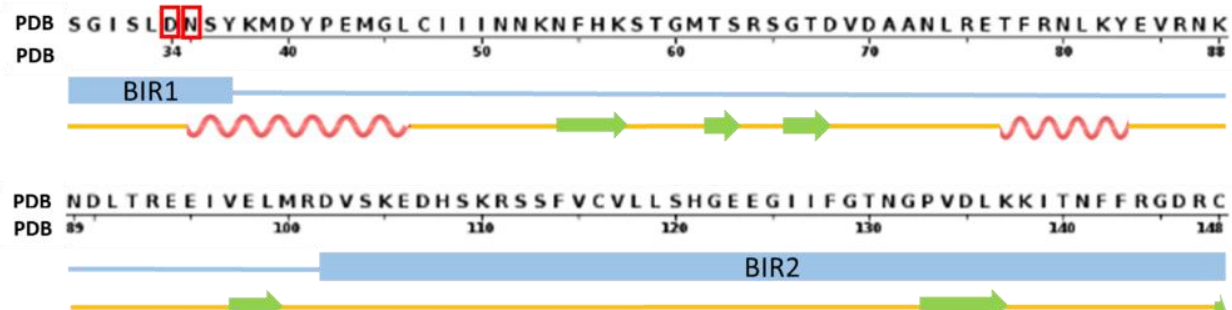

PDB RSLTGKPKLFI I QACRGTELDCG IETDSGVDDDMACHKIPVDADFLYAYSTAPGYYSWRN

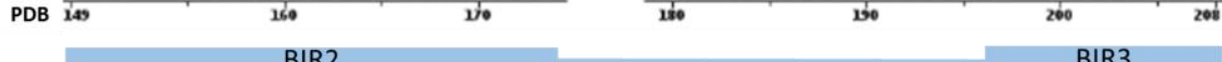

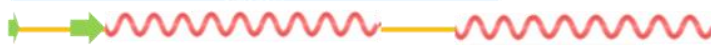
$P D B$
PDB
209 BIR3

PDB LTRELYFYH

\begin{tabular}{|c|c|c|c|c|c|c|c|c|c|c|}
\hline $\begin{array}{c}\text { Anthocyanin } \\
\text { (Ligand) }\end{array}$ & Interactin & ng site & $\begin{array}{r}\text { Amino acid } r \\
\text { Caspa }\end{array}$ & $\begin{array}{l}\text { sidues of } \\
\text { e3 }\end{array}$ & liga & ind site & $\begin{array}{c}\text { Distance } \\
\quad\left({ }^{\circ} \mathrm{A}\right)\end{array}$ & Category & Types & $\begin{array}{l}\text { Binding } \\
\text { energy } \\
\text { (cal/mol) }\end{array}$ \\
\hline \multirow{14}{*}{$\begin{array}{l}\text { Malvidin-3-O- } \\
\text { glucoside }\end{array}$} & \multirow{4}{*}{ BIR1 region } & \multirow{4}{*}{ loop } & B:ASP34:HT2 & H-Donor & $\begin{array}{l}\text { :LIG1: } \\
\mathrm{O}\end{array}$ & $\begin{array}{l}\mathrm{H}- \\
\text { Acceptc } \\
\mathrm{r}\end{array}$ & 1,80607 & Hydrogen Bond & $\begin{array}{l}\text { Conventional } \\
\text { Hydrogen Bond }\end{array}$ & \multirow{14}{*}{-304.4} \\
\hline & & & B:ASP34:OD1 & Negative & :LIG1 & $\begin{array}{l}\text { Pi- } \\
\text { Orbitals }\end{array}$ & 4,92669 & Electrostatic & Pi-Anion & \\
\hline & & & B:ASP34:0 & Steric & $\begin{array}{l}\text { :LIG1: } \\
\mathrm{O}\end{array}$ & Steric & 1,97544 & Unfavorable & $\begin{array}{l}\text { Unfavorable } \\
\text { Bump }\end{array}$ & \\
\hline & & & B:ASN35 & & & & & Van der waals & & \\
\hline & \multirow{2}{*}{\multicolumn{2}{|c|}{$\begin{array}{l}\text { BIR1-BIR2 } \\
\text { interconnection None }\end{array}$}} & & & & & & & & \\
\hline & & & A:MET222 & & & & & \multirow{3}{*}{$\begin{array}{l}\text { Van der waals } \\
\text { Van der waals } \\
\text { Van der waals }\end{array}$} & & \\
\hline & \multirow[t]{2}{*}{ BIR2 region } & \multirow[t]{2}{*}{ a-helix } & A:GLN225 & & & & & & & \\
\hline & & & A:TYR226 & & & & & & & \\
\hline & \multirow{5}{*}{$\begin{array}{l}\text { BIR2-BIR3 } \\
\text { interconnection }\end{array}$} & \multirow{5}{*}{ a-helix } & A:ARG238:NH1 & Positive & :LIG1 & $\begin{array}{l}\text { Pi- } \\
\text { Orbitals }\end{array}$ & 4,94205 & Electrostatic & Pi-Cation & \\
\hline & & & A:ARG241:NH2 & Positive & :LIG1 & $\begin{array}{l}\text { Pi- } \\
\text { Orbitals }\end{array}$ & 4,7912 & Electrostatic & Pi-Cation & \\
\hline & & & A:LYS242 & & & & & Van der waals & & \\
\hline & & & A:THR245 & & & & & Van der waals & & \\
\hline & & & A:GLU246 & & & & & Van der waals & & \\
\hline & \multicolumn{2}{|l|}{ BIR3 region } & B:LYS271 & & & & & Van der waals & & \\
\hline
\end{tabular}

Figure 3. The interaction between malvidin-3-O-glucoside and caspase-3. Overview of malvidin-3-O-glucosidecaspase-3 complex in A1, The 3D and 2D structures of malvidin-3-O-glucoside-caspase-3 complex in A2 and A3. The blue color is caspase-3 protein and the pink color is malvidin-3-O-glucoside. B. Structure-based sequence of Caspase-3 domain interacts with malvidin-3-O-glucoside. Amino acid sequence with red box indicates the binding site of anthocyanin-caspase-3. The secondary structure of caspase- 3 is represented by the green arrow ( $\beta$-sheet), the red wave ( $\alpha$-helix) and the yellow line (loop). C. The malvidin-3-O-glucoside binding site to caspase-3. 
A1

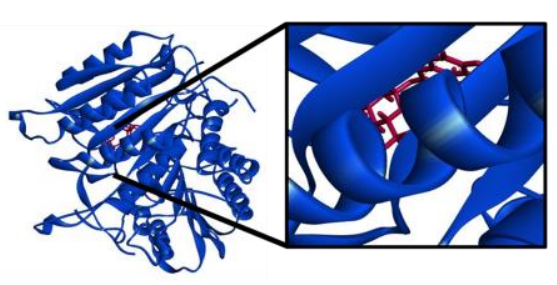

A2

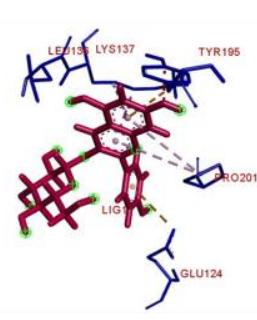

A3

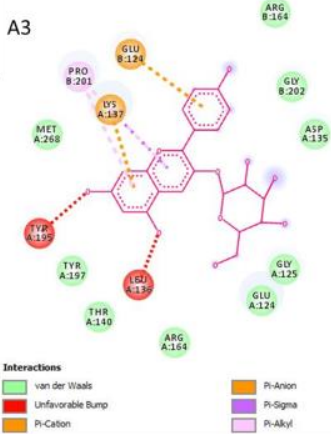

BIR1
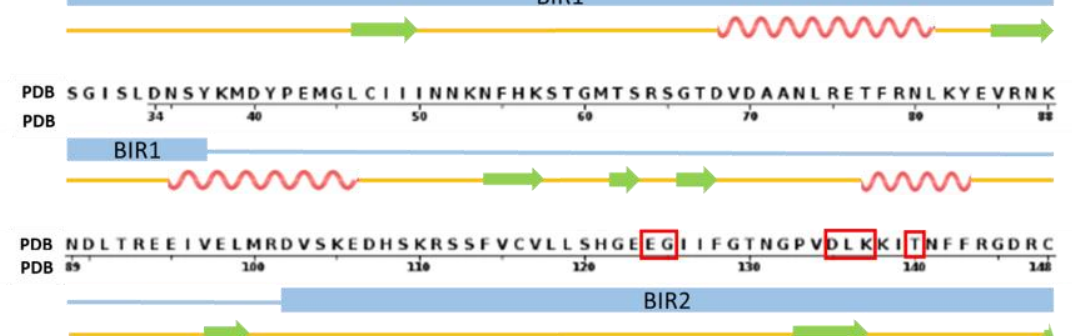

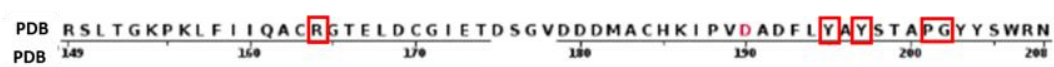
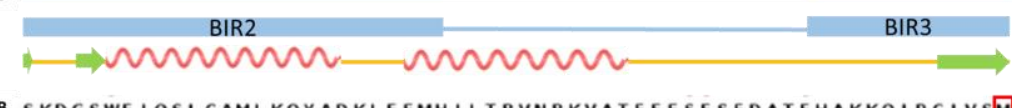

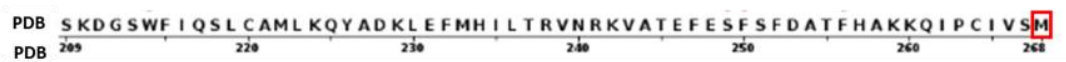

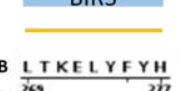

PDB LTKELYFYH

C

\begin{tabular}{|c|c|c|c|c|c|c|c|c|c|c|}
\hline \multirow[t]{3}{*}{$\begin{array}{c}\text { Anthocyanin } \\
\text { (Ligand) }\end{array}$} & \multicolumn{2}{|c|}{ Interacting site } & \multicolumn{2}{|c|}{$\begin{array}{c}\text { Amino acid residues of } \\
\text { Caspase } 3\end{array}$} & \multicolumn{2}{|c|}{ ligand site } & \multirow[t]{2}{*}{$\begin{array}{c}\text { Distance } \\
\left({ }^{\circ} \mathrm{A}\right)\end{array}$} & \multirow[t]{2}{*}{ Category } & \multirow[t]{2}{*}{ Types } & \multirow[t]{2}{*}{$\begin{array}{l}\text { Binding } \\
\text { energy } \\
\text { (cal/mol }\end{array}$} \\
\hline & BIR1 region & None & & & & & & & & \\
\hline & \multirow{9}{*}{$\begin{array}{l}\text { BIR1-BIR2 } \\
\text { interconnection }\end{array}$} & \multirow{5}{*}{ loop } & B:GLU124:OE1 & Negative & LIG1 & $\begin{array}{l}\text { Pi- } \\
\text { Orbitals }\end{array}$ & 3,92821 & Electrostatic & Pi-Anion & \multirow{18}{*}{-277.5} \\
\hline \multirow{17}{*}{$\begin{array}{l}\text { Pelargonidin-3- } \\
\text { O-glucoside }\end{array}$} & & & $\begin{array}{l}\bar{A}: G L U 124 \\
A \cdot G(Y 125\end{array}$ & & & & & \multirow{2}{*}{\multicolumn{2}{|c|}{$\begin{array}{l}\text { Van der waals } \\
\text { Van der waals } \\
\end{array}$}} & \\
\hline & & & $\frac{\text { A.GLY125 }}{\text { A:ASP135 }}$ & & & & & & & \\
\hline & & & A:LEU136:HB1 & Steric & \multicolumn{2}{|c|}{ :LIG1:OSteric } & 1,67487 & Unfavorable & $\begin{array}{l}\text { Unfavorable } \\
\text { Bump }\end{array}$ & \\
\hline & & & A:LEU136:HB1 & Steric & \multicolumn{2}{|c|}{ :LIG1:HSteric } & 1,35484 & Unfavorable & $\begin{array}{l}\text { Unfavorable } \\
\text { Bump }\end{array}$ & \\
\hline & & \multirow{4}{*}{ a-helix } & A:LYS137:NZ & Positive & :LIG1 & $\begin{array}{l}\text { Pi- } \\
\text { Orbitals }\end{array}$ & 4,89589 & Electrostatic & Pi-Cation & \\
\hline & & & A:LYS137 & Alkyl & :LIG1 & $\begin{array}{l}\text { Pi- } \\
\text { Orbitals }\end{array}$ & 4,51126 & Hydrophobic & Pi-Alkyl & \\
\hline & & & A:LYS137:CD & C-H & :LIG1 & Pi- & 3,75536 & Hydrophobic & Pi-Sigma & \\
\hline & & & $\overline{\text { A:THR140 }}$ & & & & & Van der waals & & \\
\hline & \multirow{7}{*}{ BIR2 region } & & A:ARG164 & & & & & Van der waals & & \\
\hline & & loop & B:ARG164 & & & & & Van der waals & & \\
\hline & & $\beta$-sheet & A:TYR195:CZ & Steric & :LIG1:O & OSteric & 2,17994 & Unfavorable & $\begin{array}{l}\text { Unfavorable } \\
\text { Bump }\end{array}$ & \\
\hline & & & $\overline{\text { A:TYR197 }}$ & & & & & Van der waals & & \\
\hline & & \multirow{3}{*}{ loop } & B:PRO201 & Alkyl & :LIG1 & $\begin{array}{l}\text { Pi- } \\
\text { Orbitals }\end{array}$ & 5,42826 & Hydrophobic & Pi-Alkyl & \\
\hline & & & B:PRO201 & Alkyl & :LIG1 & Pi- & 5,43589 & Hydrophobic & Pi-Alkyl & \\
\hline & & & $\overline{\text { B:GLY202 }}$ & & & & & Van der waals & & \\
\hline & $\begin{array}{l}\text { BIR2-BIR3 } \\
\text { interconnection }\end{array}$ & None & & & & & & & & \\
\hline & BIR3 region & $\beta$-sheet & A:MET268 & & & & & Van der waals & & \\
\hline
\end{tabular}

Figure 4. The interaction between pelargonidin-3-O-glucoside and caspase-3. A1. Overview of pelargonidin-3O-glucoside-caspase-3 complex, A2. The 3D structure of pelargonidin-3-O-glucoside-caspase-3 complex, A3. The 2D structure of pelargonidin-3-O-glucoside-caspase-3 complex. Caspase-3 protein and pelargonidin-3-O-glucoside are shown in blue and pink, respectively. B. Structure-based sequence of Caspase-3 domain interacts with pelargonidin-3-O-glucoside. Amino acid sequence with red box indicates the binding site of pelargonidin-3-O-glucoside-caspase-3. The secondary structure of caspase 3 was represented by the green arrow ( $\beta$-sheet), the red wave ( $\alpha$-helix) and the yellow line (loop). C. The binding site of pelargonidin-3-O-glucoside to caspase-3. 
A1

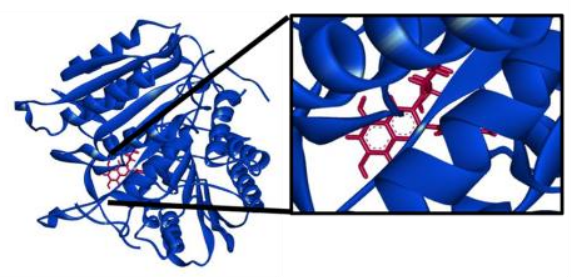

B
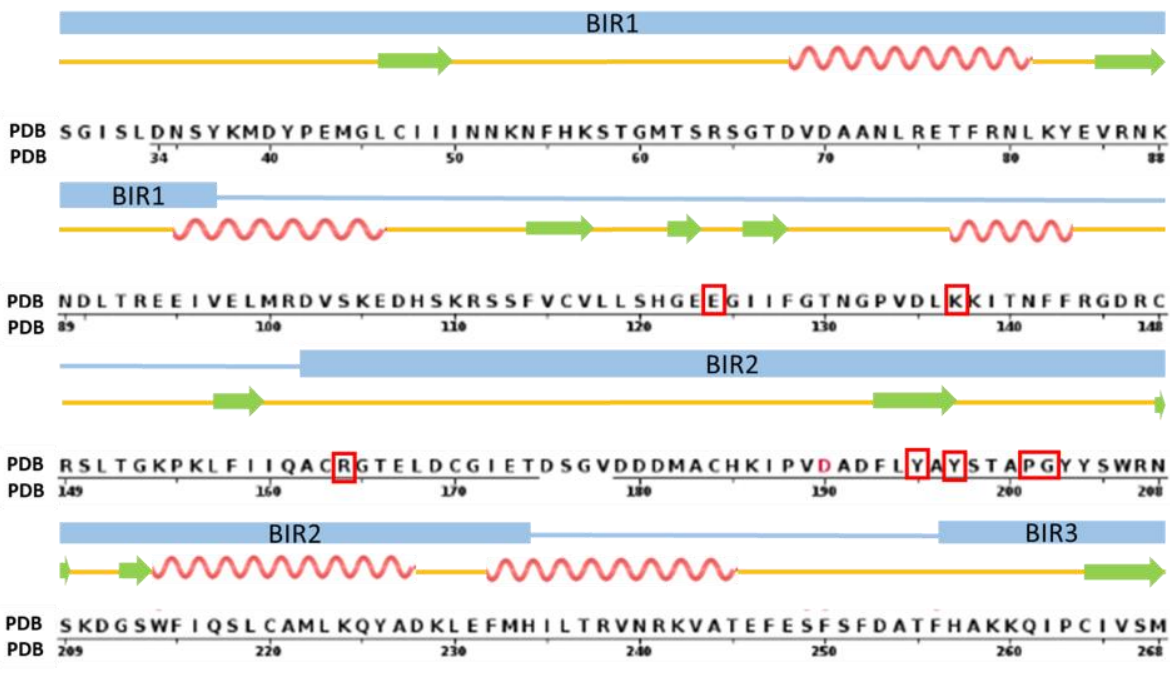

PDB 2 ios

220

230

240

250

26

BIR3

PDB LTKELYFYH

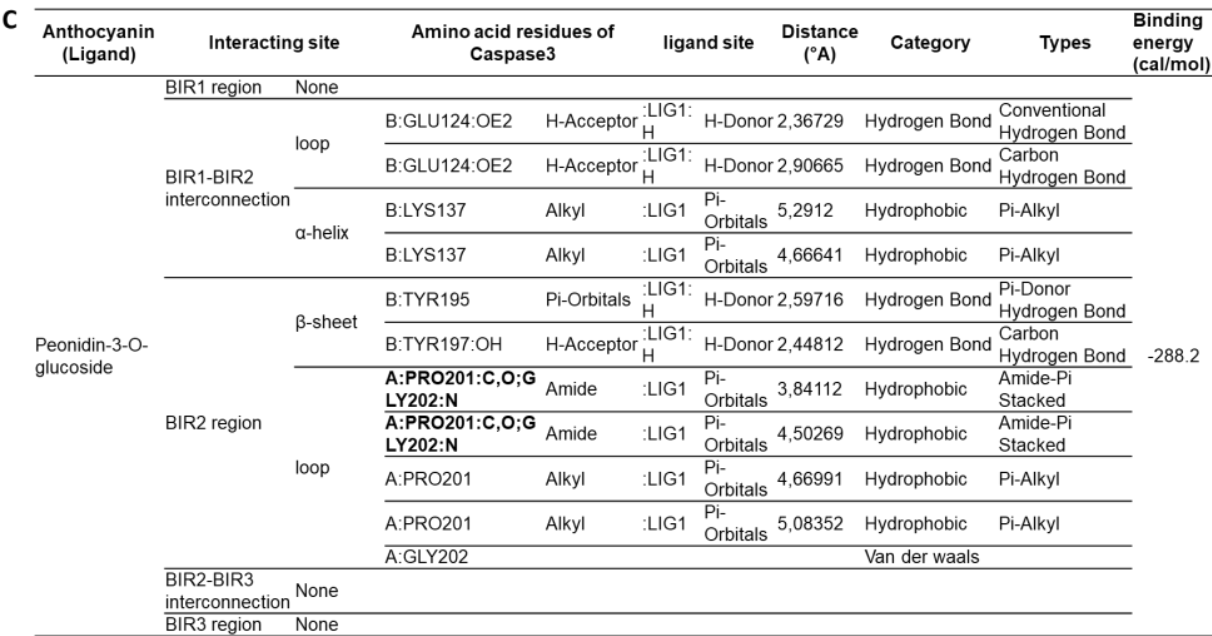

Figure 5. The interaction between peonidin-3-O-glucoside and caspase-3. A1. Overview of peonidin-3-O-glucoside-caspase-3 complex, A2. The 3D structure of caspase-3 with peonidin-3-O-glucoside complex, A3. The 2D structure of peonidin-3-O-glucoside-caspase-3 complex. Caspase-3 protein is shown in blue and peonidin-3-O-glucoside is in pink. B. Structure-based sequence of Caspase-3 domain interacts with peonidin-3-O-glucoside. Amino acid sequence with red box indicates the binding site of peonidin-3-Oglucoside-caspase-3. The secondary structure of caspase-3 is represented by the green arrow ( $\beta$-sheet), the red wave ( $\alpha$-helix) and the yellow line (loop). C. The binding site of peonidin-3-O-glucoside to caspase-3. 


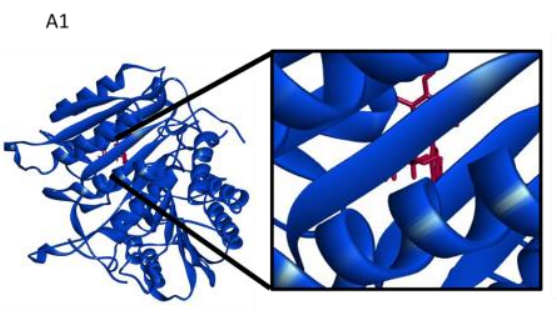

A2

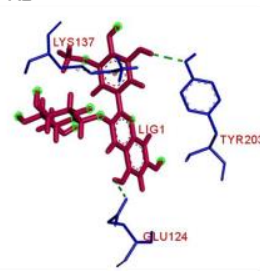

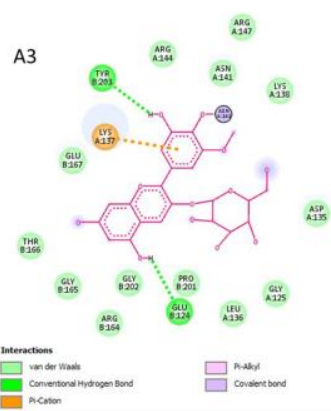

B

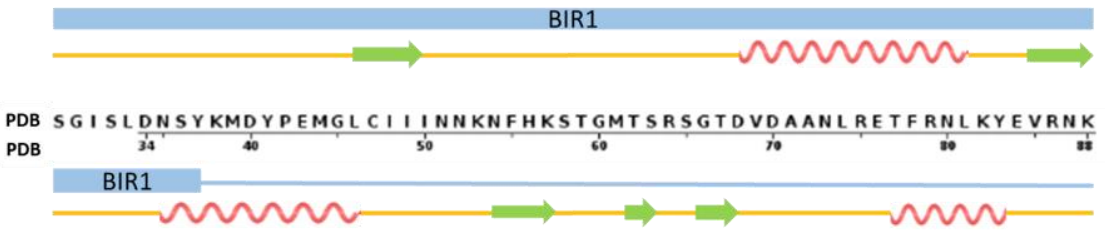

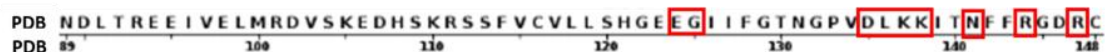
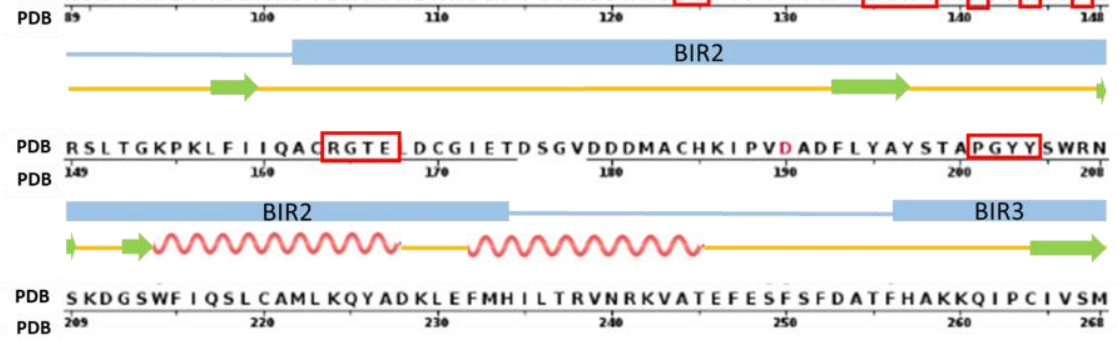

PDB 205

230

250

BIR3

PDB LTKELYFYH

PDB 26

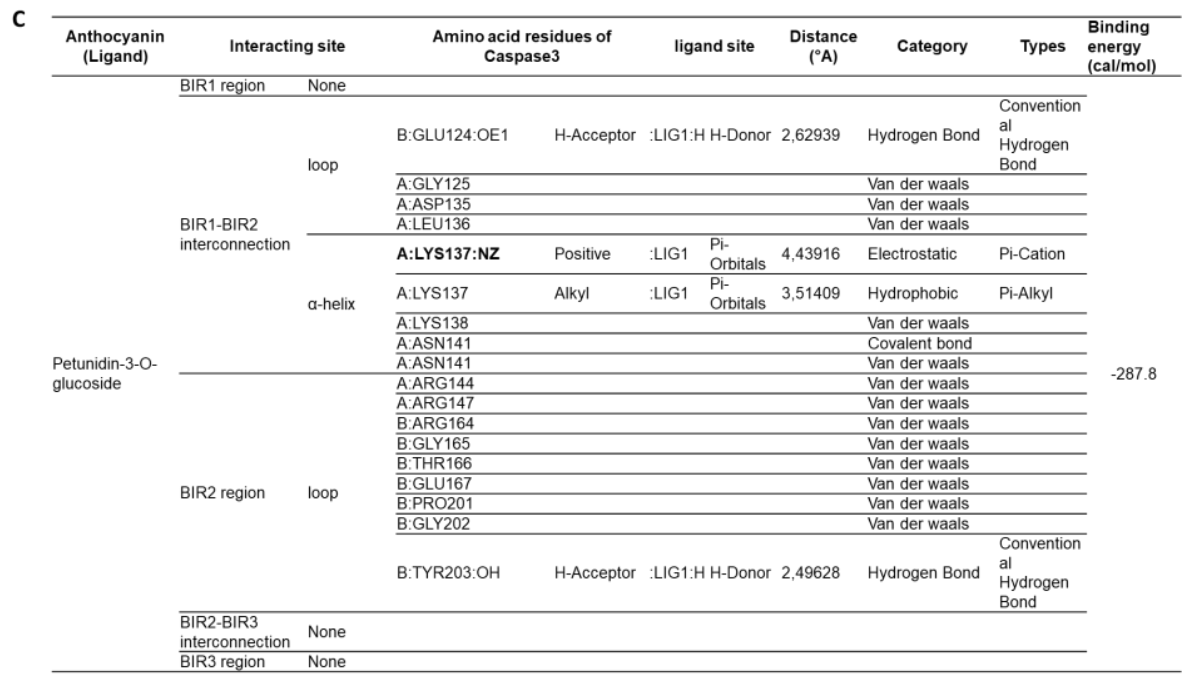

Figure 6. The interaction between petunidin-3-O-glucoside and caspase-3. The overview of the interaction, the 3D and 2D structures of petinidin-3-O-glucoside-caspase-3 complex were shown in A1, A2 and A3, respectively. The blue color as caspase-3 protein and pink color as petunidin-3-O-glucoside. The sequences and the structure of caspase- 3 are demonstrated in B. Red box indicates the active site of petunidin-3-O-glucoside on caspase-3 protein. The caspase-3 secondary structure, included $\beta$-sheet is showed by the green arrow, $\alpha$-helix in the red wave and loop structure as yellow line. C. The binding site of petunidin-3-O-glucoside to caspase-3. 
of caspase-3. Previous finding investigated the possible interaction of caspase- 3 and several natural compounds. The active sites of rosmarinic Arg341, Phe381, His237, Cys285, Ser339, Trp340, and Arg341. The curcumin-caspase-3 interaction showed some amino acid residues that were Arg179, His237, Gln283, Ala284, Tyr338, Ser339, Trp340, Arg341, Phe381 and Ser343. Luteolin interacted with the Arg179, Ser236, Gln283, Tyr338, His237, Cys285, Tyr338, Ser339, Arg341, and Phe381 of caspase-3 [13]. Our study found that all anthocyanins except malvidin-3-Oglucoside bound to caspase-3 protein in Arg164, Tyr197, and Pro201. Those binding sites were reported as the allosteric site of the interface of the caspase-3 domain. They were involved in stabilizing the active conformation of caspase- 3 as well $[29,30]$.

The anthocyanin-caspase- 3 interaction showed that anthocyanin bound to different secondary structure of caspase-3 protein (Figure 1-6 sub B and C). Cyanidin-3-O-glucoside, delphinidin-3O-glucoside, pelargonidin-3-O-glucoside, peonidin-3-O-glucoside, and petunidin-3-O-glucoside interacted with caspase-3 in BIR1-BIR2 interconnection region and BIR2 region, which the amino acid residues ranged between 98-101 and 102-234, respectively. The BIR2 region is an important region to execute the apoptotic process in the cell [8].

The active sites of anthocyanins-caspase- 3 complex were detected in $\alpha$-helix, $\beta$-sheet and loop region. Interestingly, most of the anthocyanins binding sites were located in the loop structure of caspase- 3 protein. The ligand binds to loop of caspase-3 suggested that act as inhibitor [30, 31]. The $2 \mathrm{D}$ interaction of anthocyanin-caspase- 3 performed the different kind of interactions such as hydrogen bond, hydrophobic bond, covalent bond, van der Waals, pi-alkyl, unfavorable bump and pi stacked ion. Cyanidin-3-O-glucoside and delphinidin-3-O-glucoside have only one hydrogen bond and five hydrophobic bonds. Malvidin3-O-glucoside also has only one hydrogen bond and eight amino acids with van der Waals type. Pelargonidin-3-O-glucoside shows four hydrophobic bond and nine van der Waals interaction types. Peonidin-3-O-glucoside has four hydrogen bonds and six hydrophobic bonds, while petunidin-3-O-glucoside demonstrates 13 van der Waals interaction types, two hydrogen bonds and one hydrophobic bond. Those interaction contributes the binding energy of anthocyanin-caspase-3 with the range -277.5 until $-304.4 \mathrm{cal} / \mathrm{mol}$.

The different kinds of interaction affected the binding energy free [32]. Hydrogen bonds are important contributors for the structure and interaction of protein-protein or ligand-receptor. In the drug design, hydrogen bond is critical to obtain the specificity of the drug to protein target [33-35]. In this study, malvidin-3-O-glucoside-caspase-3 interaction showed the number of hydrogen and hydrophobic bond lower than other anthocyanins, but performed the highest binding affinity. Furthermore, petunidin-3-O-glucoside- caspase-3 complex also has highest van der Waals interaction and also has one covalent bond in Asn141, which the binding energy of the complex was $287.8 \mathrm{cal} / \mathrm{mol}$. The van der Waals interaction, electrostatic and covalent bond affected the energy binding of the protein-ligand complex [32, 36-38]. Therefore, based on the interaction type, energy score and binding regions, cyanidin-3-O-glucoside, delphinidin-3-O-glucoside, pelargonidin-3O-glucoside and peonidin-3-O-glucoside are more potent as anti-apoptotic through caspase- 3 inhibition. However, other anthocyanins, malvidin-3O-glucoside and petunidin-3-O-glucoside could interact with caspase-3 and might have activity for inhibiting apoptosis.

\section{Conclusion}

This study indicated that cyanidin-3-O-glucoside, delphinidin-3-O-glucoside, pelargonidin-3O-glucoside, and peonidin-3-O-glucoside, have more potential activity as anti-apoptosis through caspase-3 interaction in BIR2 region and allosteric sites. Further experiments are necessary to develop natural compounds such as anthocyanins for inhibiting caspase-3.

\section{Acknowledgment}

This research was supported by DGHE, Ministry of Research and Technology No. 147/SP2H/LT/DRPM/2018. We gratefully acknowledge to Hazna Noor Meidinna, for english proofreading.

\section{References}

1. Rastogi RP, Richa, Sinha RP (2009) Apoptosis: Molecular mechanisms and pathogenicity. EXCLI Journal 8 155-181. doi: 10.17877/DE290R-8930. 
2. Portt L, Norman G, Clapp C et al. (2011) Biochimica et Biophysica Acta Anti-apoptosis and cell survival: A review. BBA Molecular Cell Research 1813 (1): 238-259. doi: 10.1016/j.bbamcr.2010.10.010.

3. Gordeziani M, Adamia G, Khatisashvili G, Gigolashvili G (2017) Annals of Agrarian Science Programmed cell self-liquidation ( apoptosis ). Annals of Agrarian Sciences 15 (1): 148154. doi: 10.1016/j.aasci.2016.11.001.

4. Crawford ED, Wells JA (2011) Caspase substrates and cellular remodeling. Annual Review of Biochemistry 80 (1): 10551087. doi: 10.1146/annurev-biochem-061809-121639.

5. Amor BRC, Schaub MT, Yaliraki SN, Barahona M (2016) Prediction of allosteric sites and mediating interactions through bond-to-bond propensities. Nature Communications 7 (1) 12477. doi: 10.1038/ncomms12477.

6. Tubeleviciute-Aydin A, Beautrait A, Lynham J et al. (2019) Identification of Allosteric Inhibitors against Active Caspase-6. Scientific Reports 9 (1): 1-19. doi: 10.1038/s41598-019-419307.

7. Fu SC, Imai K, Sawasaki T, Tomii K (2014) ScreenCap3: Improving prediction of caspase-3 cleavage sites using experimentally verified noncleavage sites. Proteomics 14 (17-18): 20422046. doi: 10.1002/pmic.201400002.

8. Ferreira KS, Kreutz C, MacNelly S et al. (2012) Caspase-3 feeds back on caspase-8, Bid and XIAP in type I Fas signaling in primary mouse hepatocytes. Apoptosis 17 (5): 503-515. doi: 10.1007/s10495-011-0691-0.

9. Kocab AJ, Duckett CS (2016) Inhibitor of apoptosis proteins as intracellular signaling intermediates. FEBS Journal 283 (2): 221-231. doi: 10.1111/febs.13554.

10. Sun Y, Xu Y, Geng L (2015) Caspase-3 inhibitor prevents the apoptosis of brain tissue in rats with acute cerebral infarction. Experimental and Therapeutic Medicine 10 (1): 133-138. doi: 10.3892/etm.2015.2462.

11. Shiri R, Yari F, Ahmadinejad M et al. (2014) The caspase-3 inhibitor (peptide Z-DEVD-FMK) affects the survival and function of platelets in platelet concentrate during storage. Blood Research 49 (1): 49-53. doi: 10.5045/br.2014.49.1.49.

12. Minini L, Ferraro F, Cancela S, Merlino A (2017) Insight into the mechanism of action and selectivity of caspase-3 reversible inhibitors through in silico studies. Journal of Molecular Structure 1147 558-568. doi: 10.1016/j.molstruc.2017.06.118.

13. Khan S, Ahmad K, Alshammari EMA et al. (2015) Implication of Caspase-3 as a Common Therapeutic Target for Multineurodegenerative Disorders and Its Inhibition Using Nonpeptidyl Natural Compounds. BioMed Research International 2015 1-9. doi: 10.1155/2015/379817.

14. White JB, Beckford J, Yadegarynia S et al. (2012) Some natural flavonoids are competitive inhibitors of caspase- $1,-3$, and -7 despite their cellular toxicity. Food Chemistry 131 (4): 14531459. doi: 10.1016/j.foodchem.2011.10.026.
15. Lee YM, Yoon Y, Yoon H et al. (2018) Enhanced antioxidant activity of bioactives in colored grains by nano-carriers in human lens epithelial cells. Molecules. doi: 10.3390/molecules23061327

16. Zhu F (2018) Anthocyanins in cereals: Composition and health effects. Food Research International 109 232-249. doi: 10.1016/j.foodres.2018.04.015.

17. Kawser Hossain M, Abdal Dayem A, Han J et al. (2016) Molecular mechanisms of the anti-obesity and anti-diabetic properties of flavonoids. International Journal of Molecular Sciences 17 (4): 569. doi: 10.3390/ijms17040569.

18. Li D, Wang P, Luo Y et al. (2017) Health benefits of anthocyanins and molecular mechanisms: Update from recent decade. Critical Reviews in Food Science and Nutrition 57 (8): 17291741. doi: 10.1080/10408398.2015.1030064.

19. Hosoda K, Sasahara H, Matsushita K et al. (2018) Anthocyanin and proanthocyanidin contents, antioxidant activity, and in situ degradability of black and red rice grains. Asian-Australasian Journal of Animal Sciences 31 (8): 1213-1220. doi: 10.5713/ajas.17.0655.

20. Javadzadeh Y, Hamedeyaz S (2014) Floating Drug Delivery Systems for Eradication of Helicobacter pylori in Treatment of Peptic Ulcer Disease. In: Roesler BM ed Trends Helicobacter pylori Infection. London, InTech Open. p 13.

21. Kurtys E, Eisel ULM, Hageman RJJ et al. (2018) Anti-inflammatory effects of rice bran components. Nutrition Reviews 76 (5): 372-379. doi: 10.1093/nutrit/nuy011.

22. Wang T, Li Q, Bi K (2018) Bioactive flavonoids in medicinal plants: Structure, activity and biological fate. Asian Journal of Pharmaceutical Sciences 13 (1): 12-23. doi: 10.1016/j.ajps.2017.08.004.

23. Wang E, Liu Y, Xu C, Liu J (2017) Antiproliferative and proapoptotic activities of anthocyanin and anthocyanidin extracts from blueberry fruits on B16-F10 melanoma cells. Food \& Nutrition Research 61 (1): 1325308. doi: 10.1080/16546628.2017.1325308.

24. Huang WY, Wu H, Li DJ et al. (2018) Protective Effects of Blueberry Anthocyanins against $\mathrm{H}_{2} \mathrm{O}_{2}$-Induced Oxidative Injuries in Human Retinal Pigment Epithelial Cells. Journal of Agricultural and Food Chemistry 66 (7): 1638-1648. doi: 10.1021/acs.jafc.7b06135.

25. Dallakyan S, Olson AJ (2015) Small-Molecule Library Screening by Docking with PyRx. In: Methods Mol. Biol. pp 243-250.

26. Castañeda-ovando A, Pacheco-hernández MD, Lourdes Páezhernández ME et al. (2009) Chemical studies of anthocyanins: A review Chemical studies of anthocyanins: A review. Food Chemistry 113 (4): 859-871. doi: 10.1016/j.foodchem.2008.09.001.

27. Silva S, Costa EM, Calhau C et al. (2017) Anthocyanin extraction from plant tissues: A review. Critical Reviews in Food Sci- 
ence and Nutrition 57 (14): 3072-3083. doi: 10.1080/10408398.2015.1087963.

28. Khoo HE, Azlan A, Tang ST, Lim SM (2017) Anthocyanidins and anthocyanins: colored pigments as food, pharmaceutical ingredients, and the potential health benefits. Food \& Nutrition Research $61 \quad$ (1): $1361779 . \quad$ doi: 10.1080/16546628.2017.1361779.

29. Walters J, Schipper JL, Swartz P et al. (2012) Allosteric modulation of caspase 3 through mutagenesis. Bioscience Reports 32 (4): 401-411. doi: 10.1042/BSR20120037.

30. Maciag JJ, Mackenzie SH, Tucker MB et al. (2016) Tunable allosteric library of caspase-3 identifies coupling between conserved water molecules and conformational selection. Proceedings of the National Academy of Sciences 113 (41): E6080E6088. doi: 10.1073/pnas.1603549113.

31. Vaidya S, Velázquez-Delgado EM, Abbruzzese G, Hardy JA (2011) Substrate-induced conformational changes occur in all cleaved forms of caspase-6. Journal of Molecular Biology 406 (1): 75-91. doi: 10.1016/j.jmb.2010.11.031.

32. Raharjo SJ, Mahdi C, Nurdiana N et al. (2014) Binding Energy Calculation of Patchouli Alcohol Isomer Cyclooxygenase Complexes Suggested as COX-1/COX-2 Selective Inhibitor. Advances in Bioinformatics 2014 1-12. doi: $10.1155 / 2014 / 850628$.
33. Kostal J (2016) Computational Chemistry in Predictive Toxicology. In: Fishbein J, Heilman J eds. Advances in Molecular Toxicology, 1st ed. Cambridge, Elsevier B.V. pp 139-186.

34. Chen D, Oezguen N, Urvil P et al. (2016) Regulation of proteinligand binding affinity by hydrogen bond pairing. Science Advances 2 (3): e1501240. doi: 10.1126/sciadv.1501240.

35. Menéndez CA, Accordino SR, Gerbino DC, Appignanesi GA (2016) Hydrogen Bond Dynamic Propensity Studies for Protein Binding and Drug Design. PLOS ONE 11 (10): e0165767. doi: 10.1371/journal.pone.0165767.

36. Raharjo SJ, Kikuchi T (2016) Molecular dynamic screening sesquiterpenoid pogostemon herba as suggested cyclooxygenase inhibitor. Acta Informatica Medica 24 (5): 332-337. doi: 10.5455/aim.2016.24.332-337.

37. Fatchiyah F, Rahasta AH, Cairns JRK (2017) Virtual screening and prediction of binding of caprine CSN1S2 protein tryptic peptides to glucokinase. Acta Informatica Medica 25 (4): 225231. doi: 10.5455/aim.2017.25.225-231.

38. Firdausi L, Indra MR, Fatchiyah F (2012) Binding Inhibition Between Igf1r and Igf1 by Catechin of Black Tea. Journal of Tropical Life Science 3 (2): 132-135. 
This page is intentionally left blank. 